\title{
Nosocomial Acquisition of Burkholderia gladioli Bacteremia in Two Children without Documented Cystic Fibrosis and Immunedeficiency: Case Reports
}

\author{
Nusa Matijasic ${ }^{1}$, Natasa Andrijasevic ${ }^{2}$, Amarela Lukic Grlic ${ }^{1,3}$, Iva Topalusic ${ }^{1}$, Irena Ivkovic \\ Jurekovic ${ }^{1,4}$, Marta Navratil ${ }^{1,4 *}$ \\ 1Department of Pulmonology, Allergology, Rheumatology and Clinical Immunology, Children's Hospital Zagreb, Medical School University of Zagreb, Zagreb, Croatia \\ 2Department of Microbiology, University Hospital "Sveti Duh", Zagreb, Croatia \\ 3Department of Microbiology, Children's Hospital Zagreb, Medical School University of Zagreb, Zagreb, Croatia \\ 4Faculty of Medicine, Josip Juraj Strossmayer University Osijek, Osijek, Croatia
}

Received: August 12, 2016; Accepted: September 20, 2016; Published: September 28, 2016

*Corresponding author: Marta Navratil, Department of Pulmonology, Allergology, Rheumatology and Clinical Immunology, Children's Hospital Zagreb, Klaiceva 16, 10000 Zagreb, Croatia, Tel: +385-1-4600145; Fax: +385-1-4600145; E-mail: marta.navratil@gmail.com

\begin{abstract}
Burkholderia gladioli (B. gladioli) is an environmental pathogen which causes disease in both humans and plants. B. gladioli infections in immunocompetent individuals are rare.

The following text presents two cases of nosocomial B. gladioli bacteremia in children without documented cystic fibrosis or immunedeficiency. The source of infection and route of entry are also discussed.
\end{abstract}

Keywords: Bacteremia; Burkholderia gladioli; Nosocomial; RSV

\section{Introduction}

Burkholderia gladioli (formerly known as Pseudomonas marginata) is an aerobic gram-negative rod-shaped bacteria which causes disease in both humans and plants [1]. A B. gladioli is an opportunistic pathogen in humans, associated with hospital infections that frequently colonizes respiratory tract of patients with cystic fibrosis [2-4] and Chronic Granulomatous Disease (CGD) [5-6]. The majority of publications regarding B. gladioli infections refers to immunocompromised patients: the case of B. gladioli osteomyelits in a 6-year-old Caucasian boy with CGD [7], B. gladioli pneumonia in a 55-year-old woman with AIDS [2], B. gladioli bacteriemia in a 9-month-old immunosuppressed girl with liver failure, who had previously undergone an unsuccessful liver transplantation [2], B. gladioli panbronchiolitis with bacteremia in a 62-year-old female patient with myasthenia gravis, thymoma and immune-mediated granulocytopenia treated with tacrolimus [3], B. gladioli associated abscess in a type 1 diabetic patient [8] and the case of B. gladioli bacteremia in a 54-year-old male with diabetes mellitus [9].

Contrary to this, herein we report two cases of $B$. gladioli bacteremia in immunocompetent children without documented cystic fibrosis or in immunocompetent children without documented cystic fibrosis or immunodeficiency.

\section{Case Report I}

An 18-month-old female child presented to the pediatric emergency department with dyspnea. The illness started a week before in form of cough and nasal secretion, after which she became highly febrile up to $39.5 \mathrm{C}$ with a scarce oral intake. Previously on the same day she was examined by a primary care pediatrician who introduced oral azithromycin therapy.

Her past medical history revealed an episode of mild acute bronchial obstruction in infancy, during a viral respiratory infection. Otherwise, she was a healthy child, with a positive family history for asthma.

At arrival, her general condition was impaired; she was subfebrile, with a severe tachypnea (60 breaths/min), and a heart rate of 168 beats/min. Her oxygen saturation was low, 82\%, which increased to $95 \%$ after oxygen administration (6 liters/ minute). Chest auscultation revealed diffuse expiratory wheezes and mid-inspiratory crackles of the right side.

Upon admission, a combined salbutamol and ipratropium bromide inhalation therapy was immediately started, along with intravenous corticosteroids (methylprednisolone $2 \mathrm{mg} / \mathrm{kg}$ per day) and a dual antibiotic therapy (azithromycin $10 \mathrm{mg} / \mathrm{kg}$ orally once a day for 3 days and ceftriaxone $75 \mathrm{mg} / \mathrm{kg}$ intravenously). Laboratory tests demonstrated an increased level of C-reactive protein $(97,9 \mathrm{mg} / \mathrm{L})$ and a white blood cell count (WBC) of 9400/ $\mu \mathrm{l}$, dominantly neutrophilia. A thoracic X-ray confirmed right sided, middle lobe pneumonia. Respiratory syncytial virus (RSV) was isolated in the nasopharyngeal swab.

By the third day of the hospitalization she was afebrile, respiratory stable and started to improve until the sixth day when she again became highly febrile, without an evident respiratory deterioration. Blood test revealed leukocytosis and a significant increase in inflammatory parameters (CRP 183,5 $\mathrm{mg} / \mathrm{L}$, and PCT 0,89 ng/mL), while lung ultrasonography showed 
no progression of the right sided infiltration. The treatment was modified by replacing ceftriaxone with intravenous vancomycin and meropenem. Oseltamivir was also added due to the suspected influenza infection which was subsequently excluded. Pairs of blood cultures were taken on the first and the sixth day, along with urine cultures. No bronchoalveolar lavage culture was taken. Forty eight hours after the modified therapy was introduced, the child became afebrile, and remained asymptomatic until her discharge on the day thirteen. On day eight, blood cultures taken during the clinical deterioration, came positive for Burkholderia gladioli. According to the antibiogram, the bacterium was susceptible to piperacillin-tazobactam, ceftazidime, cefepime, ciprofloxacin, amikacin, imipenem, netilmicin and meropenem. Monotherapy with meropenem was continued for the next seven days.

Due to the microbial isolate, further diagnostic work up was performed in order to excludeimmunodeficiency and cystic fibrosis. The values of chloride sweat test $(9,2 \mathrm{mmol} / \mathrm{L})$, fecal elastase $(427$ ug/g), alpha-1-antitrypsin (1,73 g/L), alpha-fetoprotein (9.5 ng/ $\mathrm{mL}$ ), immunonoglobulins (IgA 0,91 g/L, IgM 1,93 g/L, IgG 7,66 $\mathrm{g} / \mathrm{L}$ ) and respiratory burst came within referential values.

\section{Case Report Ii}

A 22-month-old female child was admitted to the Department of Gastroenterology due to eating disorder, recurrent vomiting and a poor somatic and neuro-motoric development.

She was the first child of non-consanguineous parents, with a positive family history of nutrition allergies and was living in impaired social conditions. At the age of 15 months she suffered a posttraumatic fracture of the left femur bone which was treated conservatively, but was otherwise healthy. She had been eating only inadequate portions of pureed food, and automatically vomited upon consuming any solid food, without any other signs or symptoms of a gastrointestinal disease. Upon arrival, she was hypotrophic (body weight 9400 grams - c. 3.4, BMI 13,3 kg/m²), but her general condition was not otherwise impaired. During her stay at the hospital, an extensive diagnostic work up was conducted excluding obstructive anomalies of the digestive system as a possible organic cause of vomiting. Esophagogastroduodenoscopy revealed intact mucosa, while serologic screening for celiac disease came negative. The values of chloride sweat test $(21,9 \mathrm{mmol} / \mathrm{L})$, fecal elastase (478 ug/g), and immunoglobulins (IgA 0,51 g/L, IgM $1,03 \mathrm{~g} / \mathrm{L}$, IgG 9,18 g/L) all came within referential values.

On the fifth day of hospitalization the child acquired a respiratory system infection caused by RSV, isolated in the nasopharyngeal swab. She was in good general condition, cardiopulmonary compensated, but due to a significant increase in inflammatory parameters (CRP 262,3 mg/L, PCT 3,55 ng/ $\mathrm{ml}$ ) intravenous ceftriaxone was introduced. By the third day of therapy she became afebrile, and remained that way for 24 hours, when she again became highly febrile, with signs of bacteremia and a systemic inflammatory disease. Her condition was further complicated by the left cubital vein thrombophlebitis, confirmed ultrasonically. Since the blood cultures taken upon the first peak of temperature came positive for Staphylococcus epidermidis, the treatment was modified by replacing ceftriaxone with clindamycin and co-amoxiclav. Although she reacted favorably to the above mentioned antibiotics, it was reported that gram negative bacteria were growing in the blood cultures taken during the clinical deterioration. The therapy was therefore modified once again, this time by introducing vancomycine and meropenem. Upon identification of B. gladioli, susceptible to piperacillintazobactam, ceftazidime, cefepime, ciprofloxacin, amikacin, imipenem, netilmicin and meropenem, monotherapy with meropenem was continued for a total of 10 days. Twelve hours after meropenem was introduced the girl became afebrile, and remained asymptomatic until her discharge on the day twenty six

\section{Discussion}

B. gladioli is an environmental pathogen that lives in water and soil [1]. Outbreaks of nasocomial infections may occur due to contaminated antiseptics, disinfectants, ventilators and other types of medical equipment. Moreover, person-to-person spread has been documented [10].

B. gladioli infections in immunocompetent individuals are rare, but still described in the medical literature: 63-yearold female with no prior pulmonary history who was being mechanically ventilated for complications of trauma [4], 14 cases of neonatal sepsis in hospital in Ankara, Turkey, [11], 87 cases of bacteremia in newborns in the neonatal intensive care unit in China [12], two cases of keratitis and corneal ulcers caused by B. gladioli [13].

Our first patient was a healthy 18-month-old female child with a history of wheezing episode who was not immunocomppromissed and was minimally exposed to invasive procedures during hospitalization. It is unlikely that the child had any form of pulmonary lesions similar to patients with cystic fibrosis or Chronic Obstructive Pulmonary Disease (COPD), which might have been niches for chronic colonisation and the source of infection. She had started antimicrobial treatment with commonly used broad-spectrum antibiotics (azitromicin and ceftriaxone) to which B. gladioli is intrisicly resistant, which could have also been a predisposition to infection [14]. The second patient was hypotrophic due to an inadequate caloric intake without documented immunodeficiency, cystic fibrosis or other chronic gastrointestinal or metabolic disease. In addition, both patients had confirmed $R S V$ infections that could have been a risk factor for B. gladioli infection. It is known that $R S V$ infection causes a dysregulated immune response with impaired $\mathrm{T}$ cell function as well as exaggerated inflammation via multiple mechanisms [15]. We are aware that there were no consequent positive blood cultures, which could have ruled out a probable specimen contamination. However, clinical presentation and favorable clinical respond to antibiotic treatment in both patients support our opinion that that the presented two cases were clinically significant bacteremia, rather than contamination. Certainly, these two cases of nosocomial bacteremia in the same clinic in a short time period suggest that source of infection could be contaminated medical equipment, hospital stuff or environment and less likely cross- 
infecton from other patients. These two reports provide further evidence that $B$. gladioli should be considered as an opportunistic pathogen in hospitalized immunocompetent patients undergoing minimal invasive and invasive diagnostic (venepunction) or therapeutic procedures (inhalation, parenteral therapy). Our results are in accordance with previous report of Dursun, et al. [11] and Zhou, et al. [12] where B. gladioli sepsis were mostly results of invasive procedures with low overall hospital mortality rate and $B$. gladioli infection mortality rate [11].

In our cases, the portal of entry of the pathogen is discutabile, although it is probable that it entered via patients' skin during venepunction. It is not likely that the pathogen entered through the respiratory tract by inhalation as there was no deterioration in respiratory status of both patients. Also, both patients were healthy without chronic lung disease, so there were no pulmonary lesions as possible niches for chronic colonizers and reservoir of infection. However, no bronchoalveolar lavage was done because the patients were respiratory stable and responded quickly and successfully to antibiotic therapy. In accordance with previous literature, both patients improved after In accordance with previous literature, both patients improved after appropriate antibiotic therapy, suggesting low pathogenicity of B. gladioli for otherwise healthy children of $B$. gladioli for otherwise healthy children.

\section{References}

1. Koneman's Color Atlas and Textbook of Diagnostic Microbiology. 6th Edition. By Washington Winn: Jr., Stephen Allen, William Janda, Elmer Koneman, Gary Procop, Paul Schreckenberger, and Gail Woods., Philadelphia: Lippincott Williams \& Wilkins; 2005. p. 1736.

2. Graves M, Robin T, Chipman AM, Wong J, Khashe S, Janda JM. Four additional cases of Burkholderia gladioli infection with microbiological correlates and review. Clin Infect Dis. 1997;25:838-842.

3. Imataki O, Kita N, Nakayama-Imaohji H, Kida JI, Kuwahara T, Uemura M. Bronchiolitis and bacteraemia caused by Burkholderia gladioli in a non-lung transplantation patient, New Microbes New Infect. 2014;2(6):175-6.

4. Kennedy MP, Coakley RD, Donaldson SH, Aris RM, Hohneker K, et al.
Burkholderia gladioli: Five year experience in a cystic fibrosis and lung transplantation center. J Cyst Fibros. 2007; 6(4):267-73.

5. Ross JP, Holland SM, Gill VJ, DeCarlo ES, Gallina. Severe Burkholderia (Pseudomonas) gladioli infection in chronic granulomatous disease: report of two successfully treated cases. Clin Infect Dis. 1995;21:1291-1293.

6. Greenberg DE, Goldberg JB, Stock F, Murray PR, Holland SM, and LiPuma JJ. Recurrent Burkholderia Infection in Patients with Chronic Granulomatous Disease: 11-Year Experience at a Large Referral Center. Clin Infect Dis. 2009;48 (11):1577-1579.

7. Boyanton B, Noroski L, Reddy H, Dishop M, Hicks M, Versalovic J, et al. Burkholderia gladioli osteomyelitis in association with chronic granulomatous disease: case report and review. Pediatr. Infect. Dis.J. 2005;24: 837-839.

8. Waseem M, Al-Sherbeeni S, Al-Malki MH, Al-Ghamdi MS. Burkholderia gladioli associated abscess in a type 1 diabetic patient. Saudi Med J. 2008;29(7):1048-50.

9. Shin JH, Kim SH, Shin MG, Suh SP, Ryang DW, Jeong $\mathrm{MH}$ Bacteremia due to Burkholderia gladioli: case report. Clin Infect Dis. 1997;25(5):1264-5.

10. Wilsher ML, Kolbe J, Morris AJ, Welch DF. Nosocomial acquisition of Burkholderia gladioli in patients with cystic fibrosis. Am J Respir Critir Care Med. 1997;155(4):1436-40.

11. Dursun A, Zenciroglu A, Karagol BS, Hakan N, Okumus N, Gol N, Tanir G. Burkholderia gladioli sepsis in newborns. Eur J Pediatr. 2012;171(10):1503-9.

12. Zhou F, Ning $\mathrm{H}$, Chen F, Wu W, Chen A, Zhang J. Burkholderia gladioli infection isolated from the blood cultures of newborns in the neonatal intensive care unit. Eur J Clin Microbiol Infect Dis. 2015;34(8):1533-7.

13. Lestin F, Kraak R, Podbielski A. Two cases of keratitis and corneal ulcers caused by Burkholderia gladioli. J Clin Microbiol. 2008;46(7):2445-9.

14. Matthaiou DK, Chasou E, Atmatzidis S, Tsolkas P. A case of bacteremia due to Burkholderia cepacia in a patient without cystic fibrosis. Respiratory Medicine CME. 2011;4:144-145.

15. Openshaw PJ, Chiu C. Protective and dysregulated T cell immunity in RSV infection. Curr Opin Virol. 2013;3(4):468-74.

16. Gharib A, Gupta S (2016) Skeletal and Joint Manifestations of Primary Immunodeficiency Diseases. SOJ Immunol 4(1): 1-13. DOI:http://dx.doi.org/10.15226/2372-0948/4/1/00145 